\title{
Response to Pegylated Interferon Plus Ribavirin in Patients with Hepatitis C Virus Genotype 6a Infection from Guangdong and Guangxi Province of China
}

\author{
Wangxia Tong, ${ }^{1}$ Jianyun Zhu, ${ }^{2}$ Ning Luo, ${ }^{1}$ Xiaohua Yang, ${ }^{2}$ Zhiying Lei, ${ }^{2}$ \\ Xiaoliang Huang, ${ }^{3}$ Zhixin Zhao, ${ }^{2}$ Xiaohong Zhang, ${ }^{2}$ Zhiliang Gao, ${ }^{2}$ and Zhonghua Jiang ${ }^{4}$ \\ ${ }^{1}$ Ruikang Hospital Affiliated to Guangxi University of Chinese Medicine, Nanning 530000, China \\ ${ }^{2}$ Department of Infectious Diseases, the Third Affiliated Hospital of Sun Yat-Sen University, Guangzhou 510080, China \\ ${ }^{3}$ Pingguo County People's Hospital, Guangxi 531400, China \\ ${ }^{4}$ Department of Emergency, Affiliated Liuzhou Hospital of Guangxi Traditional Chinese Medicine University, Guangxi 545007, China \\ Correspondence should be addressed to Wangxia Tong; twx_01@126.com
}

Received 18 July 2015; Revised 31 October 2015; Accepted 4 November 2015

Academic Editor: Paolo Gionchetti

Copyright (C) 2016 Wangxia Tong et al. This is an open access article distributed under the Creative Commons Attribution License, which permits unrestricted use, distribution, and reproduction in any medium, provided the original work is properly cited.

\begin{abstract}
Aim. Our aim is to survey the treatment effect of PEG-IFN plus ribavirin in patients infected with HCV genotype 6a in Guangdong and Guangxi province of China and investigate best course of antiviral treatment for patients with HCV-6a infection. Methods. 515 eligible patients received subcutaneous $180 \mu \mathrm{g}$ PEG-IFN $\alpha$-2a or $1.5 \mu \mathrm{g} / \mathrm{kg}$ PEG-IFN $\alpha$-2b once weekly plus oral ribavirin. Primary outcome was SVR by intention-to-treat analysis. Secondary outcome was RVR, cEVR, ETR, and relapse rate. Results. SVR in patients with HCV-6a infection treated for 48 weeks was comparable to that in patients with HCV-2/3 infection $(80.9 \%$ versus $82.5 \%$, $p=0.812)$ and higher than that in patients with HCV-1b infection $(80.9 \%$ versus $67.2 \%, p=0.014)$. ETR $(98.9 \%$ versus $90.6 \%$, $p=0.016)$, virological response at month 3 of end-of- treatment $(88.8 \%$ versus $76.6 \%, p=0.044)$, SVR $(80.9 \%$ versus $65.6 \%$, $p=0.032)$, and virological response at month 12 of end-of-treatment $(76.4 \%$ versus $60.9 \%, p=0.04)$ in patients with HCV-6a infection treated for 48 weeks were higher than those in patients with HCV-6a infection treated for 24 weeks. Conclusion. SVR in patients with HCV-6a treated for 48 weeks was comparable to that in patients with HCV-2/3 infection and higher than that in patients with HCV-1b infection; patients with HCV-6a infection treated for 48 weeks had a superior treatment response than patients treated for 24 weeks.
\end{abstract}

\section{Introduction}

Although the treatment of hepatitis $\mathrm{C}$ virus (HCV) has made significant advances with development of nucleotide polymerase inhibitors antivirals currently [1], PEG-IFN and ribaviron-based therapy will still be the mainstay of HCV treatment in China Mainland in the near future, because most new medications may be either unavailable or unaffordable. According to the mass of clinical reports, the therapeutic effect of pegylated interferon (PEG-IFN) and ribavirin is related to various factors, including genotype, age, treatment adherence, and RVR, where genotype is the most important one $[2,3]$. HCV is classified into 6 genotypes and more than 90 subtypes and shows diverse patterns of geographic distribution $[4,5]$. HCV-1b was still the most widely distributed genotype in China Mainland, followed by 2a, 6a, $3 a$, and $3 b[6,7]$. In accordance with Gu et al. study in 2013 [8], genotype $6 \mathrm{a}$ has displaced genotype $2 \mathrm{a}$ and became the second genotype in Pearl River Delta China. For antiviral treatment course of PEG-IFN plus ribavirin for $\mathrm{CHC}$ patients infected with HCV-6a in China Mainland, the Chinese physicians have often taken the recommended 48-week course adopted by patients infected HCV-1b [9]. It is debatable whether it is suitable for CHC patients infected with HCV6a in China Mainland. After all, the treatment guideline of 48 weeks is made for patients infected with HCV-lb. According 
to few clinical report regarding treatment for $\mathrm{CHC}$ patients infected with genotype $6[10,11]$, duration of 24 weeks therapy for patients with RVR appeared equally effective as 48 weeks of therapy. And we want to know whether 24 weeks could be standard duration for CHC patients infected with genotype 6a. The data about antiviral therapy for $\mathrm{CHC}$ patients in China Mainland are sparse and these experiments are often restricted by the amount of samples and lack of representation [11]. Therefore, future research needs to be based upon more large survey sample in representative regions. The objective of this study is to research the therapeutic effect of PEGIFN and ribavirin combination for $\mathrm{CHC}$ patients with various genotypes in Guangdong and Guangxi province of China and explore the best duration of treatment for $\mathrm{CHC}$ patients infected with genotype 6 .

\section{Objects and Method}

2.1. Patients. CHC patients with HCV-RNA positive are from the division of infectious diseases at the third affiliated hospital, Sun Yat-Sen University and Hepatology Center of Ruikang Hospital Affiliated to Guangxi University of Chinese Medicine, between December 2012 and June 2015. Routinely, a simple blood test is used to screen the patients for chronic liver disease. The latter refers to the fact that during the past six months or for a longer period ALT (alanine aminotransferase) elevation $(>30 \mathrm{U} / \mathrm{L})$ in serum is repeatedly or persistently tested. All eligible patients are enrolled in a registry and created follow-up records. The inclusion criteria included (1) presence of anti-HCV antibody (Roche Diagnostics, Branchburg, New Jersey, USA), (2) detectable serum HCV RNA determined by real-time RTPCR analysis (COBAS Amplicor HCV Monitor Test, v2.0; Roche Diagnostics, Branchburg, NJ; limit of quantitation $600 \mathrm{IU} / \mathrm{mL}$ ) for $>6$ months, (3) HCV genotypes 1b, 6a, and $2 / 3$ determined by nucleotide sequencing of $\mathrm{E} 1$ and NS5B region followed by phylogenetic analysis, (4) elevated alanine aminotransferase (ALT) levels 3 times greater than the upper limit of normal, and (5) histological features of chronic inflammation in the past 3 months. The patients were excluded if they had prior antiviral treatment. Further exclusion criteria included (1) anemia (male $\mathrm{Hb}<13 \mathrm{~g} / \mathrm{dL}$, female $\mathrm{Hb}<12 \mathrm{~g} / \mathrm{dL}$ ), (2) neutropenia (neutrophil count $<1500$ count $/ \mathrm{mm}^{3}$ ), (3) thrombocytopenia (blood platelet count $<70,000$ count $/ \mathrm{mm}^{3}$ ), (4) coinfection with hepatitis $B$ virus or human immunodeficiency virus, (5) coinfection with HCV nongenotypes $1 \mathrm{~b}, 6 \mathrm{a}$, and $2 / 3$, (6) combined with other serious complications, (7) alcoholism (alcohol intake $>20 \mathrm{~g} / \mathrm{d}$ ), (8) drug addicts, (9) uncontrolled psychiatric or autoimmune disease, and (10) pregnancy and lactation.

2.2. Design. The eligible objects was offered prescribed course of treatment according to different HCV genotypes, 48 weeks for genotype $1 b$, and 24 weeks for genotype $2 / 3[3,12]$, 48 and 24 weeks for genotype 6 a respectively [13]. Patients were treated with either PEG-IFN $\alpha$-2a (180 $\mu \mathrm{g}$ subcutaneously once weekly) or PEG-IFN $\alpha-2 b(1.5 \mu \mathrm{g} / \mathrm{kg} /$ week subcutaneously) in combination with oral ribavirin (a weightbased dose, $15 \mathrm{mg} / \mathrm{kg} /$ day). The patients received their first and second PEG-IFN treatment in inpatient clinics for two weeks in order to observe serious side effects. Then, patients are scheduled to outpatient hospital departments for subsequent antiviral treatment and had at least a 6-month follow-up period after the end of treatment.

2.3. Follow-Up. No patients had reduced drug doses. Patients were evaluated clinically along with laboratory testing (including complete blood cell count, serum ALT and AST level, serum albumin level, and bilirubin level), at entry, every 4 weeks during the treatment, and every 12 weeks after endof-treatment, to assess efficacy and safety of treatment. Serum HCV RNA levels were measured at baseline, week 4, week 12, end-of-treatment, and week 24 after end-of-treatment. HCV genotyping was performed at baseline by nucleotide sequencing, genotypes were determined by phylogenic analysis using NS5B and E1 sequences [14]. The assessment of degree of liver fibrosis was done by the noninvasive method of transient elastography (Fibroscan). Based on Lemoine et al. report [15], we selected the cut point of $\geq 8.7 \mathrm{kpa}$ for $\geq \mathrm{F} 2$ stage fibrosis, the cut point of $\geq 9.5 \mathrm{kpa}$ for $\geq \mathrm{F} 3$ stage fibrosis, and the cut point of $\geq 14.5 \mathrm{kpa}$ for $\geq \mathrm{F} 4$ stage fibrosis.

2.4. Ethics. The study was approved by hospital ethics committee and all patients provided written informed consent.

2.5. Efficacy Assessment. The primary outcome of efficacy was SVR (sustained virologic response), defined as an undetectable serum HCV RNA level at week 24 after treatment. RVR (rapid virologic response) was defined as an undetectable serum HCV RNA level at week 4 of therapy. cEVR (complete early virologic response) was defined as an undetectable serum HCV RNA level at week 12 of therapy. ETR (end-of-treatment response) was defined as an undetectable serum HCV RNA level at end-of-treatment. The patients dropped out of study were considered as achieving a SVR unsuccessfully. Relapse was defined as achieving ETR but becoming positive for HCV RNA after the cessation of treatment. VR $2 \mathrm{~W}$ (virological response at week 2 of therapy) is defined as an undetectable serum HCV RNA level at week 2 of therapy. VR $3 \mathrm{M}$ (virological response at month 3 of endof-treatment) is defined as an undetectable serum HCV RNA level at month 3 of end-of-treatment. VR $12 \mathrm{M}$ (virological response at month 12 of end-of-treatment) is defined as an undetectable serum HCV RNA level at month 12 of end-oftreatment.

2.6. Statistical Analysis. Ranked data by Rank-Sum test was used to compare continuous variables if tests normality is observed. Chi-squared tests were used to compare categorical variables across the different genotype groups. The relatedness of potential treatment predictors to SVR was analyzed by univariate and multivariate logistic regression analyses. Statistical significance was defined as a two-side $p$ value of 0.05 or less. All analyses were performed using a statistical software package (SPSS, version 16.0; Chicago, IL, USA). 
TABLE 1: The baseline characteristics of patients with different HCV genotype infection.

\begin{tabular}{|c|c|c|c|c|c|}
\hline \multirow{2}{*}{ Characteristics } & \multicolumn{2}{|c|}{ HCV-6a $(n=153)$} & \multirow{2}{*}{$\mathrm{HCV}-2 / 3(n=103)$} & \multirow{2}{*}{ HCV-1b $(n=259)$} & \multirow{2}{*}{$p$ value $^{*}$} \\
\hline & $(24 \mathrm{w}, n=64)$ & $(48 \mathrm{w}, n=89)$ & & & \\
\hline Age (years) & $34.2 \pm 11.6$ & $36.6 \pm 10.6$ & $36.5 \pm 14.06$ & $36.7 \pm 13.03$ & 0.639 \\
\hline BMI & $22.0 \pm 4.0$ & $22.1 \pm 3.9$ & $22.1 \pm 2.62$ & $22.4 \pm 3.93$ & 0.36 \\
\hline Male $(n)$ & $40(62.5 \%)$ & $59(63.3 \%)$ & $62(60.2 \%)$ & $163(62.9 \%)$ & 0.857 \\
\hline $\operatorname{IDU}(n)^{\dagger}$ & $17(26.6 \%)$ & $25(28.1 \%)$ & $14(13.6 \%)$ & $24(9.3 \%)$ & $<0.001$ \\
\hline Blood transfusion $(n)$ & $15(23.4 \%)$ & $21(23.6 \%)$ & $37(35.9 \%)$ & $149(57.5 \%)$ & $<0.001$ \\
\hline Family history of HCC $(n)^{\dagger}$ & $4(6.2 \%)$ & $6(6.7 \%)$ & $4(3.9 \%)$ & $7(2.7 \%)$ & 0.302 \\
\hline PEG-IFN $\alpha-2 \mathrm{a}(n)$ & $50(78.1 \%)$ & $67(75.3 \%)$ & $85(82.5 \%)$ & $223(86.1 \%)$ & 0.094 \\
\hline $\operatorname{ALT}(\mathrm{U} / \mathrm{L})$ & $82.9 \pm 8.1$ & $80.0 \pm 6.9$ & $87.9 \pm 3.0$ & $78.1 \pm 9.0$ & 0.955 \\
\hline HCV RNA $\left({ }_{\log } \mathrm{IU} / \mathrm{mL}\right)$ & $6.1 \pm 1.0$ & $6.2 \pm 1.1$ & $5.6 \pm 1.1$ & $6.4 \pm 0.9$ & 0.106 \\
\hline Cirrhosis $(n)$ & $6(9.4 \%)$ & $5(5.6 \%)$ & $9(8.7 \%)$ & $37(14.3 \%)$ & 0.118 \\
\hline
\end{tabular}

BMI, body mass index; ${ }^{\dagger}$ IDU, intravenous drug use; HCC, hepatocellular carcinoma; ALT, alanine aminotransferase; HCV RNA, hepatitis C virus ribonucleic acid; cirrhosis, the cut point of $\geq 14.5 \mathrm{kpa}$ for $\geq \mathrm{F} 4$ stage fibrosis; ${ }^{*} p$ value comparing the different genotype groups.

\section{Results}

3.1. Patient Characteristics. We collected data from $1342 \mathrm{CHC}$ patients infected with genotype $1 \mathrm{~b}$ or $2 / 3$ or $6 \mathrm{a}$. The HCV isolates were genotyped by means of a phylogenetic analysis of the E1 and/or NS5B sequences with references representing various genotypes and subtypes. According to inclusion and exclusion criteria, 515 patients were included in final analysis. We identified 153 patients with HCV genotype 6a (64 patients for 24 weeks and 89 patients for 48 weeks), 259 patients with HCV genotype $1 b$, and 103 patients with HCV genotype $2 / 3$. Table 1 describes the demographic and baseline laboratory characteristics of patients among different genotype groups (including age, BMI, gender, IDU, blood transfusion, family history of HCC, type of PEG-IFN, ALT, HCV RNA, and cirrhosis). Patients infected with HCV genotype 6a (27.5\%) included more intravenous drug users than those infected with other genotype groups $(p=0.001)$. The proportion of patients through blood transfusion in HCV genotype $1 \mathrm{~b}$ (57.5\%) was higher than other genotype groups $(p<0.001)$. Age $(p=0.639)$, gender $(p=0.857)$, BMI $(p=0.36)$, family history of HCC $(p=0.302)$, type of PEG-IFN ( $p=0.094)$, and cirrhosis $(p=0.118)$ in four groups were not significant.

3.2. Comparison of Virological Response among Patients with HCV-6a Infection Treated for 48 Weeks, HCV-2/3, and HCV-1b Infection. The RVR, cEVR, ETR, and SVR rate were showed in Figure 1. Patients with HCV-6a infection treated for 48 weeks had a higher ETR rate than patients with $\mathrm{HCV}-2 / 3$ infection $(98.9 \%$ versus $92.2 \%, p=0.03)$. RVR rate $(88.8 \%$ versus $95.1 \%, p>0.05)$, cEVR rate $(98.9 \%$ versus $99.0 \%, p>$ $0.05)$ and SVR rate $(80.9 \%$ versus $82.5 \%, p>0.05)$ in patients with HCV-6a infection treated for 48 weeks were comparable to those in patients with $\mathrm{HCV}-2 / 3$ infection. RVR rate $(88.8 \%$ versus $76.4 \%, p=0.013)$, cEVR rate $(98.9 \%$ versus $93.1 \%$, $p=0.037)$, ETR rate $(98.9 \%$ versus $93.4 \%, p=0.046)$, and SVR rate $(80.9 \%$ versus $67.2 \%, p=0.014)$ in patients with HCV-6a infection treated for 48 weeks were higher than those in patients with HCV-1b infection. Although ETR rate in patients with $\mathrm{HCV}-2 / 3$ infection was comparable to that

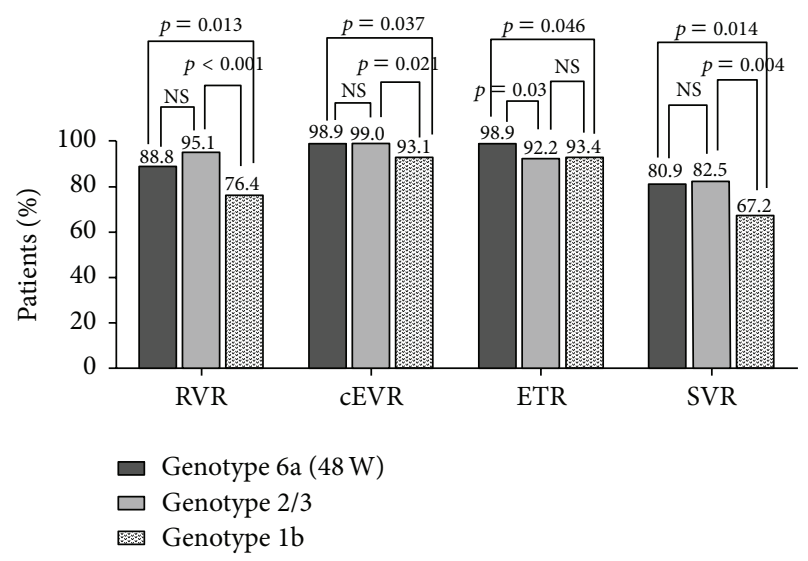

FIGURE 1: Comparison of the virological response rate to therapy with PEG-IFN plus ribavirin among patients with HCV-6a infection treated for 48 weeks, HCV-2/3, HCV-1b infection. RVR, rapid virologic response; cEVR, complete early virologic response; ETR, end-of-treatment response; SVR, sustained virologic response.

in patients with HCV-1b infection ( $p=0.684)$, RVR rate, cEVR rate, and SVR rate in patients with HCV-2/3 infection were higher than those in patients with HCV-1b infection $(p<0.05)$. The rate of relapse in patients infected with HCV-1b (23.6\%) was higher than that in patients infected with HCV-6a treated for 48 weeks $(15.3 \%, p=0.236)$ and HCV-2/3 (9.2\%, $p=0.018)$ during 6-month follow-up after end-of-treatment. The rate of relapse in patients with HCV6a infection treated for 48 weeks was comparable to that in patients with HCV-2/3 infection ( $p=0.298$ ).

3.3. Comparison of Virological Response between Patients with HCV-6a Infection Treated for 48 Weeks and 24 Weeks. We compared virological response between patients infected with HCV-6a treated for 48 weeks and patients infected with HCV-6a treated for 24 weeks, and RVR, cEVR, ETR, and SVR rate were showed in Figure 2. Virological response at week 2 of therapy $(p=0.429)$, RVR $(p=0.482)$, and 


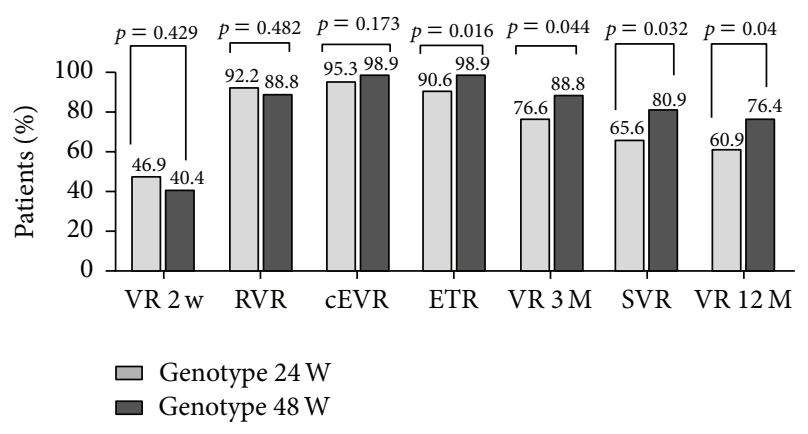

FIgURE 2: Comparison of the virological response rate between patients with HCV-6a infection treated for 48 weeks and 24 weeks. VR $2 \mathrm{~W}$, virological response at week 2 of therapy; VR $3 \mathrm{M}$, virological response at month 3 of end-of-treatment; VR $12 \mathrm{M}$, virological response at month 12 of end-of-treatment. RVR, rapid virologic response; cEVR, complete early virologic response; ETR, end-of-treatment response; SVR, sustained virologic response.

cEVR $(p=0.173)$ in patients with HCV-6a infection treated for 48 weeks were similar to those in patients with HCV6a infection treated for 24 weeks. ETR rate $(98.9 \%$ versus $90.6 \%, p=0.016)$, virological response at month 3 of endof-treatment $(88.8 \%$ versus $76.6 \%, p=0.044)$, SVR $(80.9 \%$ versus $65.6 \%, p=0.032$ ), and virological response at month 12 of end-of-treatment $(76.4 \%$ versus $60.9 \%, p=0.04)$ in patients with HCV-6a infection treated for 48 weeks were higher than those in patients with HCV-6a infection treated for 24 weeks. The rate of relapse and drop-out rate in patients with HCV-6a infection treated for 48 weeks were comparable to those in patients with HCV-6a infection treated for 24 weeks $(p>0.05)$.

The trend graph on virological response to combined therapy among patients with different HCV genotype infections was showed in Figure 3. For patients with HCV-6a infection treated for 48 weeks and patients with HCV-1b infection, virological response rate increased rapidly from week 2 of therapy (RVR) to week 12 of therapy (cEVR), virological response rate was basically flat from week 12 of therapy (cEVR) to end-of-treatment (ETR), and virological response rate was trending downward from end-of-treatment (ETR) to month 6 of end-of-treatment (SVR). For patients with HCV-6a infection treated for 24 weeks and patients with HCV-2/3 infection, virological response rate increased slowly from week 2 of therapy (RVR) to week 12 of therapy (cEVR), virological response rate decreased slowly from week 12 of therapy (cEVR) to end-of-treatment (ETR), and there was no an obvious plateau from cEVR to ETR. Virological response rate was trending downward from end-of-treatment (ETR) to month 6 of end-of-treatment (SVR).

3.4. Predictive Factors Associated with SVR in Patients with $H C V-6 a$ Infection. Potential predictors of SVR in patients with HCV-6a infection including age, gender, BMI, cirrhosis, ALT level, HCV RNA level, genotypes, cEVR, and RVR were examined. In univariate analysis, age $(\mathrm{OR}=4.136,95 \%$ CI: $1.240-13.802, p=0.021)$, cirrhosis $(\mathrm{OR}=7.038,95 \%$ CI: $1.119-44.256, p=0.038)$, and RVR (OR $=5.798,95 \%$
CI: 2.056-16.354, $p=0.001$ ) were associated with SVR. In multivariate analysis to identify predictor of SVR in patients with HCV-6a infection, the independent factor associated with SVR was age (OR $=5.772,95 \%$ CI: 1.153-28.898, $p=$ $0.033)$.

\section{Discussion}

In this study, we researched effect of PEG-IFN plus ribavirin therapy in $515 \mathrm{CHC}$ patients with different genotype in Guangdong and Guangxi province of China. We found that SVR in patients with HCV-6a infection treated for 48 weeks was comparable to that in patients with $\mathrm{HCV}-2 / 3$ infection and higher than that in patients with HCV-1b infection; patients with HCV-6a infection treated for 48 weeks had a superior treatment response than patients treated for 24 weeks. For patients with HCV-6a infection, age of the subjects was significantly associated with SVR.

This study is the largest population-based retrospective research about therapeutic effects of PEG-IFN plus ribavirin on CHC patients from China Mainland so far and focuses on HCV genotype 6a mainly. The patients (patients infected with HCV-6a mainly) in this study came from the southern coastal region of China Mainland (Guangdong and Guangxi provinces). Guangdong province, home to the Pearl River Delta, is one of the most important trade and financial center in China, Guangxi Zhuang Autonomous Region, located in the southwest coastal region of China, adjoined with Southeast Asian nations. Due to their special geographical situation and the complexity and variety of HCV genotype distribution, the two provinces have the representation in pattern of HCV genotypes in Southern China.

24 weeks of PEG-IFN plus ribavirin therapy were the internationally accepted standard treatment duration for HCV-2/3, and SVR in patients with HCV genotype 2/3 in Asia region was $75 \%-100 \%$. The rate of SVR in patients with HCV genotype $2 / 3(82.5 \%)$ in this study falls in line with that in most other reports $(75 \%-100 \%)[16,17]$.

Although ETR was superior in patients with HCV-6a infection treated for 48 weeks versus HCV-2/3 infection ( $p=$ 0.03 ). There were no significant differences in RVR, cEVR, and SVR in patients with HCV-6a infection treated for 48 weeks and patients with $\mathrm{HCV}-2 / 3$ infection $(p>0.05)$. The rate of relapse in patients with HCV-6a infection treated for 48 weeks was comparable to that in patients with $\mathrm{HCV}-2 / 3$ infection $(p=0.298)$. The results demonstrated that patients with HCV-6a infection treated with PEG-IFN and ribavirin combination treatment for 48 weeks have similar effect as patients with $\mathrm{HCV}-2 / 3$. And the finding in our study was consistent with the result as Nguyen et al. reported [13].

Our surveys showed that SVR in patients with HCV-1b infection $(67.2 \%)$ is comparable to what has been reported by other Asian area studies (59\%-76) [18-20]. RVR, cEVR, ETR, and SVR were inferior in patients with HCV-1b infection versus patients with HCV-6a treated with 48 weeks ( $p<$ 0.05). Although ETR in patients with HCV-2/3 infection were no different than that in patients with HCV-1b infection, RVR, cEVR, and SVR in patients with HCV-2/3 infection were higher than those in patients with HCV-1b infection 


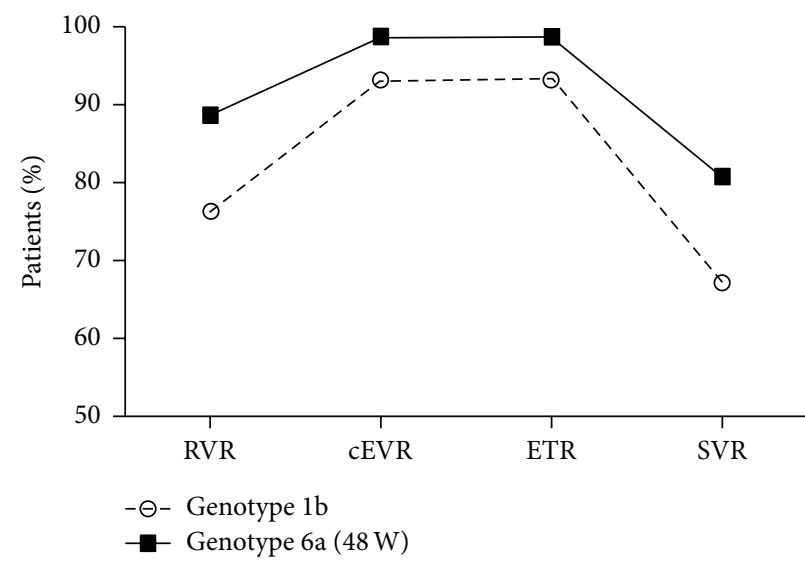

(a)

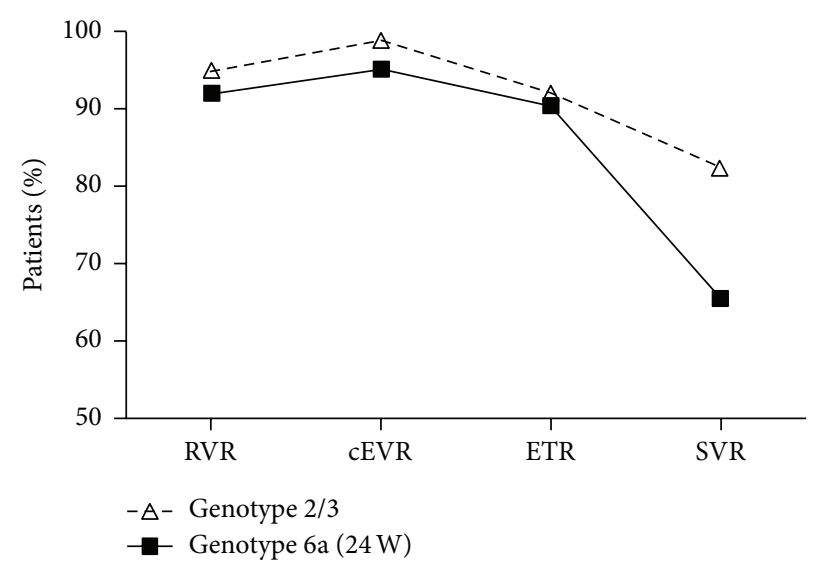

(b)

FIGURE 3: The trend graph of virological response to combined therapy among patients with HCV-1b and HCV-6a-48W (a), HCV-2/3 and HCV-6a-24W (b). RVR, rapid virologic response; cEVR, complete early virologic response; ETR, end-of-treatment response; SVR, sustained virologic response.

$(p<0.05)$. The rate of relapse in patients with HCV-1b infection was highest. These survey results indicated that patients with HCV-1b infection treated with PEG-IFN and ribavirin combination treatment have worse effect.

As had been reported by Zhou and Thu earlier [10, 11], they believed patients with HCV-6a treated with PEGIFN and ribavirin combination treatment for 24 weeks have similar effect as patients with HCV-6a treated for 48 weeks. Our results showed that SVR was inferior in patients with HCV-6a infection treated for 24 weeks versus 48 weeks ( $p=$ $0.032)$. Virological response at week 2 of therapy $(p=0.429)$, $\operatorname{RVR}(p=0.482)$, and cEVR $(p=0.173)$ in patients with HCV-6a infection treated for 48 weeks were similar to those in patients with HCV-6a infection treated for 24 weeks, so there was no significant difference between the two groups above. But ETR rate $(p=0.016)$, virological response at month 3 of end-of-treatment ( $p=0.044)$, SVR $(p=0.032)$, and virological response at month 12 of end-of-treatment $(p=0.04)$ in patients with HCV-6a infection treated for 48 weeks were higher than those in patients with HCV6a infection treated for 24 weeks, and there was significant difference between the two groups above. What drives the differences? According to our analysis, it is the course of treatment. Time span was 12 weeks from week 12 of therapy (EVR) to end-of-treatment (ETR) for patients with HCV-6a infection treated for 24 weeks and 36 weeks for patients with HCV-6a infection treated for 48 weeks. We can think that the difference in virologic response between two treatment groups appeared to be due to difference in time length of therapy. The possibility of difference in the demographic and baseline laboratory characteristics and drop-out rate was ruled out, because there was no significant difference in the characteristic between two groups.

In accordance with the trend graph of virological response to combined therapy among patients with different HCV genotype infections, a line of rise first then fall was presented on HCV-6a (48 weeks) group and HCV-1b (48 weeks) group, there was an obvious plateau in the middle of the line, and the trend graph was trapezoid-shaped. We think the excellent effect in treatment can be maintained by longerlasting therapy and manifested as the plateau. A trend graph of rise first then fall was also presented on HCV-6a (24 weeks) group and HCV-2/3 (24 weeks) group, but there was not an obvious plateau. Virological response at week 12 of therapy (cEVR) was the highest point. We can consider that shortterm combined treatment (24 weeks) was inferior to longerterm treatment (48 weeks) for patients with HCV-6a infected.

The weakness in the study was retrospective design, and there was sample selection bias in patients with HCV6a infection treated for 24 weeks and 48 weeks. What is important is that the sample size was small for patients with HCV-6a infection. Future researches need to be based upon more large survey HCV-6a samples and randomized controlled trials.

\section{Conclusion}

SVR in patients with HCV-6a infection treated for 48 weeks was comparable to that in patients with $\mathrm{HCV}-2 / 3$ infection and higher than that in patients with HCV-1b infection; patients with HCV-6a infection treated for 48 weeks had a superior treatment response than patients treated for 24 weeks. For patients with HCV-6a infection, age of the subjects was significantly associated with SVR.

\section{Abbreviations}

PEG-IFN: Pegylated interferon

HCV: $\quad$ Hepatitis $\mathrm{C}$ virus

SVR: $\quad$ Sustained virologic response

RVR: $\quad$ Rapid virologic response

cEVR: Complete early virologic response

ETR: $\quad$ End-of-treatment response

CHC: $\quad$ Chronic hepatitis $\mathrm{C}$

HCV-RNA: Hepatitis C virus ribonucleic acid

ALT: $\quad$ Alanine aminotransferase 
$\begin{array}{ll}\text { RT-PCR: } & \text { Reverse transcriptase-polymerase chain } \\ & \text { reaction } \\ \text { BMI: } & \text { Body mass index } \\ \text { HCC: } & \text { Hepatocellular carcinoma. }\end{array}$

\section{Conflict of Interests}

The authors declare that there is no conflict of interests regarding the publication of this paper.

\section{Authors' Contribution}

Jianyun Zhu equally contributed as a first author.

\section{Acknowledgments}

Appreciation is expressed to Dr. Qingshou Sheng, Professor Shengyuan Yu, and Professor Ruozhuo Liu for their assistance in the research.

\section{References}

[1] S. Kumar and I. M. Jacobson, "Antiviral therapy with nucleotide polymerase inhibitors for chronic hepatitis C," Journal of Hepatology, vol. 61, no. 1, pp. S91-S97, 2014.

[2] F. Poordad, K. R. Reddy, and P. Martin, "Rapid virologic response: a new milestone in the management of chronic hepatitis C," Clinical Infectious Diseases, vol. 46, no. 1, pp. 7884, 2008.

[3] C. H. Liu, C. J. Liu, C. L. Lin et al., "Pegylated interferon-alpha2a plus ribavirin for treatment-naive Asian patients with hepatitis $\mathrm{C}$ virus genotype 1 infection: a multicenter, randomized controlled trial," Clinical Infectious Diseases, vol. 47, no. 10, pp. 1260-1269, 2008.

[4] P. Simmonds, J. Bukh, C. Combet et al., "Consensus proposals for a unified system of nomenclature of hepatitis $\mathrm{C}$ virus genotypes," Hepatology, vol. 42, no. 4, pp. 962-973, 2005.

[5] V. H. Pham, H. D. P. Nguyen, P. T. Ho et al., "Very high prevalence of hepatitis $C$ virus genotype 6 variants in southern Vietnam: large-scale survey based on sequence determination," Japanese Journal of Infectious Diseases, vol. 64, no. 6, pp. 537539, 2011.

[6] L. Lu, M. Wang, W. Xia et al., "Migration patterns of hepatitis $\mathrm{C}$ virus in China characterized for five major subtypes based on samples from 411 volunteer blood donors from 17 provinces and municipalities," Journal of Virology, vol. 88, no. 13, pp. 71207129, 2014.

[7] X. Rong, R. Xu, H. Xiong et al., "Increased prevalence of hepatitis $\mathrm{C}$ virus subtype $6 \mathrm{a}$ in China: a comparison between 2004-2007 and 2008-2011," Archives of Virology, vol. 159, no. 12, pp. 3231-3237, 2014.

[8] L. Gu, W. Tong, M. Yuan, T. Lu, C. Li, and L. Lu, "An increased diversity of $\mathrm{HCV}$ isolates were characterized among 393 patients with liver disease in China representing six genotypes, 12 subtypes, and two novel genotype 6 variants," Journal of Clinical Virology, vol. 57, no. 4, pp. 311-317, 2013.

[9] G. W. McCaughan, M. Omata, D. Amarapurkar et al., "Asian Pacific Association for the Study of the Liver consensus statements on the diagnosis, management and treatment of hepatitis C virus infection," Journal of Gastroenterology and Hepatology, vol. 22, no. 5, pp. 615-633, 2007.
[10] P. T. Thu Thuy, C. Bunchorntavakul, H. Tan Dat, and K. Rajender Reddy, "A randomized trial of 48 versus 24 weeks of combination pegylated interferon and ribavirin therapy in genotype 6 chronic hepatitis C," Journal of Hepatology, vol. 56, no. 5, pp. 1012-1018, 2012.

[11] Y. Q. Zhou, X. H. Wang, G. H. Hong et al., "Twenty-four weeks of pegylated interferon plus ribavirin effectively treat patients with HCV genotype 6a," Journal of Viral Hepatitis, vol. 18, no. 8, pp. 595-600, 2011.

[12] A. Mangia, R. Santoro, N. Minerva et al., "Peginterferon alfa-2b and ribavirin for 12 vs. 24 weeks in HCV genotype 2 or 3," The New England Journal of Medicine, vol. 352, no. 25, pp. 2609-2617, 2005.

[13] N. H. Nguyen, P. VuTien, R. T. Garcia et al., "Response to pegylated interferon and ribavirin in Asian American patients with chronic hepatitis C genotypes 1 vs $2 / 3$ vs 6," Journal of Viral Hepatitis, vol. 17, no. 10, pp. 691-697, 2010.

[14] L. Lu, T. Nakano, Y. He, Y. Fu, C. H. Hagedorn, and B. $\mathrm{H}$. Robertson, "Hepatitis $\mathrm{C}$ virus genotype distribution in China: predominance of closely related subtype $1 \mathrm{~b}$ isolates and existence of new genotype 6 variants," Journal of Medical Virology, vol. 75, no. 4, pp. 538-549, 2005.

[15] M. Lemoine, S. Katsahian, M. Ziol et al., "Liver stiffness measurement as a predictive tool of clinically significant portal hypertension in patients with compensated hepatitis $C$ virus or alcohol-related cirrhosis," Alimentary Pharmacology and Therapeutics, vol. 28, no. 9, pp. 1102-1110, 2008.

[16] A. Mangia, F. Bandiera, G. Montalto et al., "Individualized treatment with combination of Peg-interferon alpha $2 \mathrm{~b}$ and ribavirin in patients infected with HCV genotype 3," Journal of Hepatology, vol. 53, no. 6, pp. 1000-1005, 2010.

[17] O. Dalgard, K. Bjoro, H. Ring-Larsen, and H. Verbaan, "In patients with HCV genotype 2 or 3 infection and RVR 14 weeks treatment is noninferior to 24 weeks. Pooled analysis of two Scandinavian trials," European Journal of Gastroenterology and Hepatology, vol. 22, no. 5, pp. 552-556, 2010.

[18] S. Bruno, C. Cammà, V. Di Marco et al., "Peginterferon alfa-2b plus ribavirin for naïve patients with genotype 1 chronic hepatitis C: a randomized controlled trial," Journal of Hepatology, vol. 41, no. 3, pp. 474-481, 2004.

[19] S. Missiha, J. Heathcote, T. Arenovich, K. Khan, and The Canadian Pegasys Expanded Access Group, "Impact of Asian race on response to combination therapy with peginterferon alfa-2a and ribavirin in chronic hepatitis C," The American Journal of Gastroenterology, vol. 102, no. 10, pp. 2181-2188, 2007.

[20] K. K. Yan, M. Guirgis, T. Dinh et al., "Treatment responses in Asians and Caucasians with chronic hepatitis C infection," World Journal of Gastroenterology, vol. 14, no. 21, pp. 3416-3420, 2008 . 


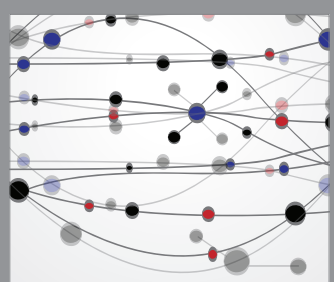

The Scientific World Journal
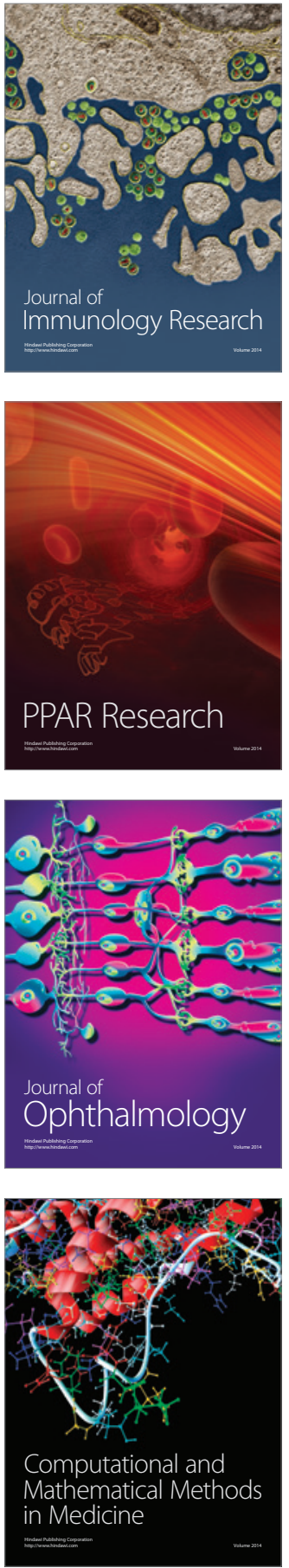

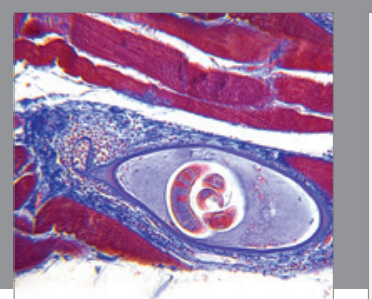

Gastroenterology Research and Practice

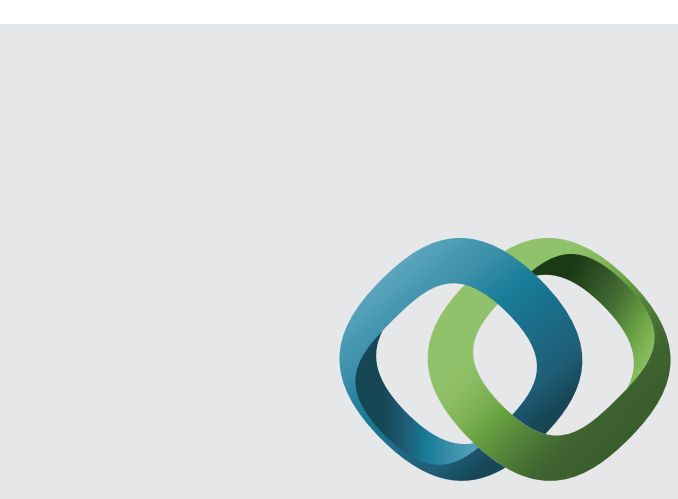

\section{Hindawi}

Submit your manuscripts at

http://www.hindawi.com
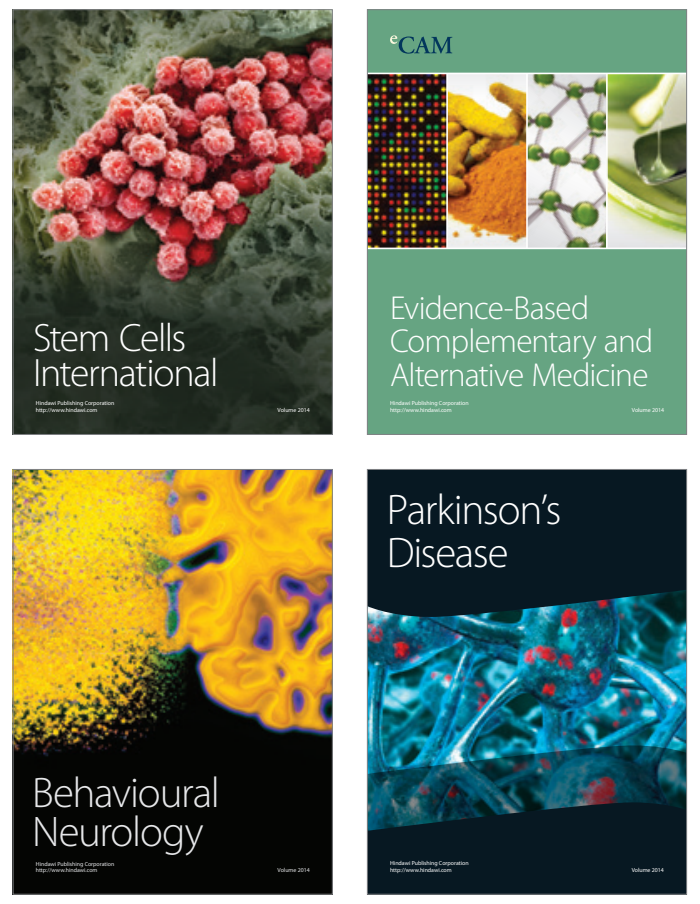
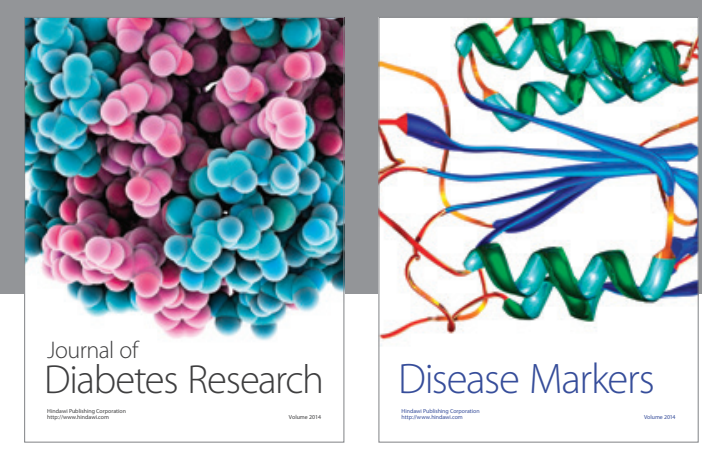

Disease Markers
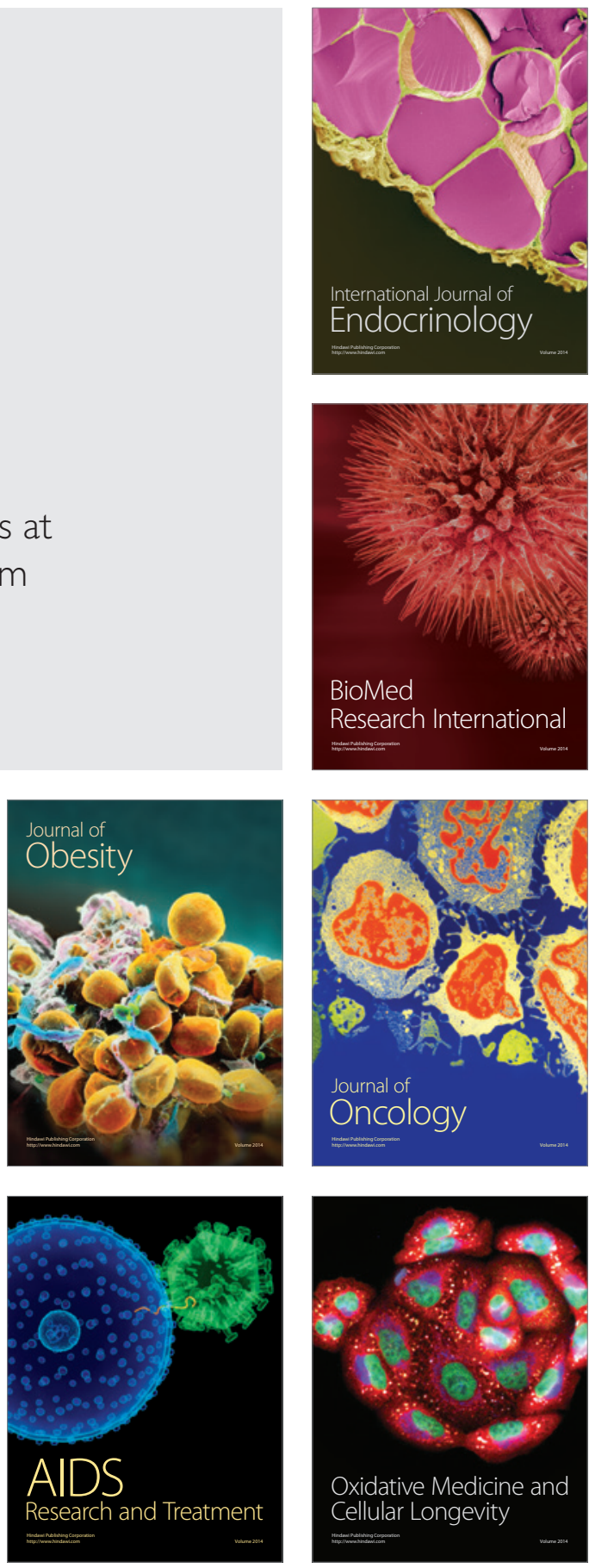